\title{
New Books
}

W. A. S e x t o n, Chemical Constitution and Biological Activity. (Industrial Chemistry Series). E. and F. N. Spon, Ltd., London, 1949. 55s., pp. XXI + 412.

This work is divided into two sections, of which the first deals with the chemical compounds and processes that play a part in biological activity in general; the second part deals with selected subjects chosen, as far as possible, with a view of providing illustrations for the principles outlined in the earlier chapters. The latter part includes various growth factors, choline and its derivatives, cancer problems, antigens and antibodies.

In one of the first chapters the author declares that biological activity "must be considered as a function of the molecule as a whole". One feels grateful for this clear formulation of an extremely important principle, which is often being sinned against by other writers in this field. One further notes with satisfaction that the author presents in detail the results of Ferguson's investigations on general narcotics; as shown by Ferguson, the thermodynamic activity corresponding to the minimal effective concentrations of the narcotics is approximately constant. The author is exceptionally well-read and gives a great number of references. It is a surprise, however, that so few general views are given concerning the main theme, which is the connection of chemical constitution with physiological effect. Many pharmacodynamically related groups of substances have by now been arranged in well-defined structure schemes, e.g. analgetics, histaminolytics, antispasmodics, local anesthetics and curare-active sub- stances. The author treats these fields very superficially. - One looks in vain for a mention of the great importance of the amino group for drugs; the part of the book which deals with basic groups consists of only 20 lines. - As an example for the isosteric principle the author mentions, inter alia, morphine and pethidine, but as the author does not treat analgetics in greater detail, the reader may easily get the impression that these drugs are dependent on a morphine-like steric configuration; This is of course not the case. - Further the author seems rather uncritical when he completely accepts some questionable concepts concerning the role of acetylcholine in the activity of the nervous system. - The parts of the book dealing with vitamins, hormones, enzymes and cancerogenic compounds are treated very thoroughly and in great detail.

Despite the inadequacies mentioned above this book has so many definite merits that it may be warmly recommen. ded. The material treated is very extensive and the author has made a consistent effort to cite the sources. The author's good stylistic ability should also contribute to its success.

Nils Löfgren

R. H. F. $M$ anske and H. L. $\mathrm{H}$ ol$\mathrm{mes}$. The Alkaloids. Chemistry and Physiology. Volume I. Academic Press Inc., New York, 1950. viii +525 pp. $\$ 10.00$.

This is the first volume of a series of five (according to the preface by the editors) which will gather together "all the 
pertinent knowledge of the chemistry and pharmacology of the alkaloids". The pharmacological aspects of the alkaloids, the chemistry of which are so thoroughly described in this volume, is missing. It is therefore to be supposed that one of the following volumes will deal with these aspects of the subject. The physiology of the alkaloids, that is alkaloid formation and metabolism in plants, is dealt with in one of the chapters in the present volume. According to the statement given in the preface the sub-title should include "Pharmacology" instead of "Physiology".

One of the editors, R. H. F. Manske, is the author of the first Chapter: Sources of alkaloids and their isolation (11 pp.). It gives a short introduction to the natural occurrence of alkaloids and their distribution in the plant. It also contains general remarks on isolation, separation, and purification. The second editor, H. L. Holmes, writes about the tropane alkaloids (97 pp.) and the strychnos alkaloids (140 pp.). Both chapters represent excellent reviews of the pure chemistry of these groups of alkaloids. They contain several clearly arranged charts illustrating the relationship of transformation products of the better known substances. The accompanying tables of physical constants for the alkaloids and their transformation products are most valuable. The table for the tropane alkaloids occupies 54 pp., and for the strychnos alkaloids 57 pp. Especially noteworthy is the section on the elucidation of the structure of strychnine and brucine, although the recent contribution of Robinson (1947-1948) to the constitution of strychnine, brucine, and vomicine has not been considered. Generally, the authors of the various chapters have considered the literature up to 1946-1947.
The editors, however, have planned to issue supplements at periodic intervals.

W. O. James has contributed with a fine chapter (75 pp.) on the biological aspects of the alkaloids in the plant. He gives an account of the biosynthesis of alkaloids, considering the site of alkaloid formation and the factors affecting it, in addition to the distribution of alkaloids in various physiological parts of the plant and alkaloids in grafts.

L. Marion writes two chapters, one on the comparatively few naturally occurring pyrrolidine alkaloids (17 pp.), and the other on the pyridine alkaloids (105 pp.). The latter compounds are described in the following order: the alkaloids of pepper, the areca nut, trigonelline, pomegranate root bark, Lobelia, Ricinus, Leucaena, poison hemlock, tobacco, and of Ammodendron. The chapter constitutes a fine survey on the chemistry of all these compounds and is accompanied by a comprehensive list of references (518 ref.).

Another chapter (59 pp.) by N.J. Leonard deals with the Senecio alkaloids. Similar alkaloids in the Heliotropium, Trachelanthus, and Trichodesma genera and in Crotalaria plants are also described here. All these compounds, the chemistry of which has been elucidated during the last 20 years, are closely related alkaloids, most of them alkamine esters with only one nitrogen atom.

An author index and a comprehensive subject index make the book easy to use as a "Handbuch".

Altogether this first volume is a valuable contribution to the ever growing literature in the chemical field of natural products. It will be exceedingly helpful to all working with alkaloids, chemists as well as pharmacologists.

Klas-Bertil Augustinsson 